\title{
Bleb Morphology Assessment and Imaging
}

\author{
${ }^{1}$ Mandeep Singh, ${ }^{2}$ Paul TK Chew \\ ${ }^{1}$ Department of Ophthalmology, National University Hospital, Singapore \\ ${ }^{2}$ Department of Ophthalmology, Yong Loo Lin School of Medicine, National University of Singapore, Singapore
}

\section{INTRODUCTION}

Trabeculectomy has been widely applied as treatment for glaucoma since its introduction in $1968 .^{1}$ The aim of trabeculectomy is to lower intraocular pressure (IOP) in order to slow the progression of glaucomatous optic neuropathy and visual loss. ${ }^{2,3}$

The outcome of this surgery depends upon the formation of a functioning shunt to enable egress of aqueous from the eye, leading to the development of a filtration bleb. The development of the bleb is crucial for long-term surgical success, and is affected by wound healing characteristics of the eye. Scarring of structures at the trabeculectomy site including conjunctiva, episclera, scleral flap and ostium may lead to poor formation of the bleb and hence poor shunt function resulting in suboptimal IOP control.

Trabeculectomy blebs have been imaged using a range of imaging modalities in an effort to characterize bleb morphology in relation to function. Here we present a review of bleb imaging techniques and their potential uses in bleb morphology evaluation and classification.

\section{RELEVANCE OF BLEB MORPHOLOGY}

\section{Function}

Bleb morphology after trabeculectomy is an important clinical parameter; a careful evaluation of bleb morphology in the early postoperative period may reveal features that predict functional outcome, i.e. success or failure. ${ }^{4}$ Examples of blebs are shown in Figures 1A to D.

In an early study, Vesti classified blebs into three morphological types and found different rates of success. ${ }^{5}$ Blebs were grouped into ‘diffuse blebs', 'flap-sized blebs' and 'no bleb' groups, and success rates of $92 \%, 64 \%$ and $43 \%$ were found in each group respectively. It is interesting to note that in this report a successful outcome was found a significant number of eyes in which there was no bleb; it is possible that some of these blebs may have had a degree of bleb wall thickening that was too small to be appreciated clinically, and which may be more apparent using current imaging techniques as are described below.
Shingleton correlated morphological features found in blebs to the level of IOP. ${ }^{6}$ In this study it was reported that bleb injection, large and ropelike vessels, thickening of the bleb wall, localized filtering blebs and high-domed blebs were early signs of failure. In contrast, diffuse blebs, paucity of vessels, a quiet surface, elevated blebs and microcystic changes in the conjunctiva were found to correlate with good IOP control.

Picht et al in 1997 evaluated five morphological features of blebs, including presence of microcysts, quantity of conjunctival vessels, shape of conjunctival vessels, presence of encapsulation and height of the bleb, to identify features that correlate with trabeculectomy success or failure. ${ }^{7}$ They found that a higher quantity of microcysts, lower quantity of conjunctival vessels and cork screw vessels, lower prevalence of encapsulation and low bleb height correlated with a favorable outcome.

Early features of blebs have been described which have predictive value in terms of future functional outcome. Sacu et al in 2003 showed that classification of filtering blebs after trabeculectomy with MMC in the early postoperative period may help to disclose patients with an increased failure risk. ${ }^{8}$ In this study, eyes with conjunctival subepithelial microcysts observed in the first 2 postoperative weeks had significantly lower mean IOP than eyes without. Eyes with corkscrew vessels in the first 2 weeks had poorer outcomes.

\section{Complications}

Bleb leak may lead to the development of postoperative hypotony. The use of antimetabolites such as mitomycin- $\mathrm{C}$ (MMC) has been associated with bleb leak. ${ }^{9-12} \mathrm{Hu}$ et al reported that bleb leaks were more likely to be found in blebs with large avascular areas. ${ }^{13}$ Thin walled blebs have also been found to be associated with bleb leakage. ${ }^{14,15}$

Bleb leakage has been found to be a risk factor for the development of blebitis and bleb-related endophthalmitis. ${ }^{16-20}$ These complications could lead to a devastating outcome for the patient; evaluation of bleb morphology with regards to features that predict bleb leak may allow the clinician to anticipate these complications and manage the bleb accordingly. 

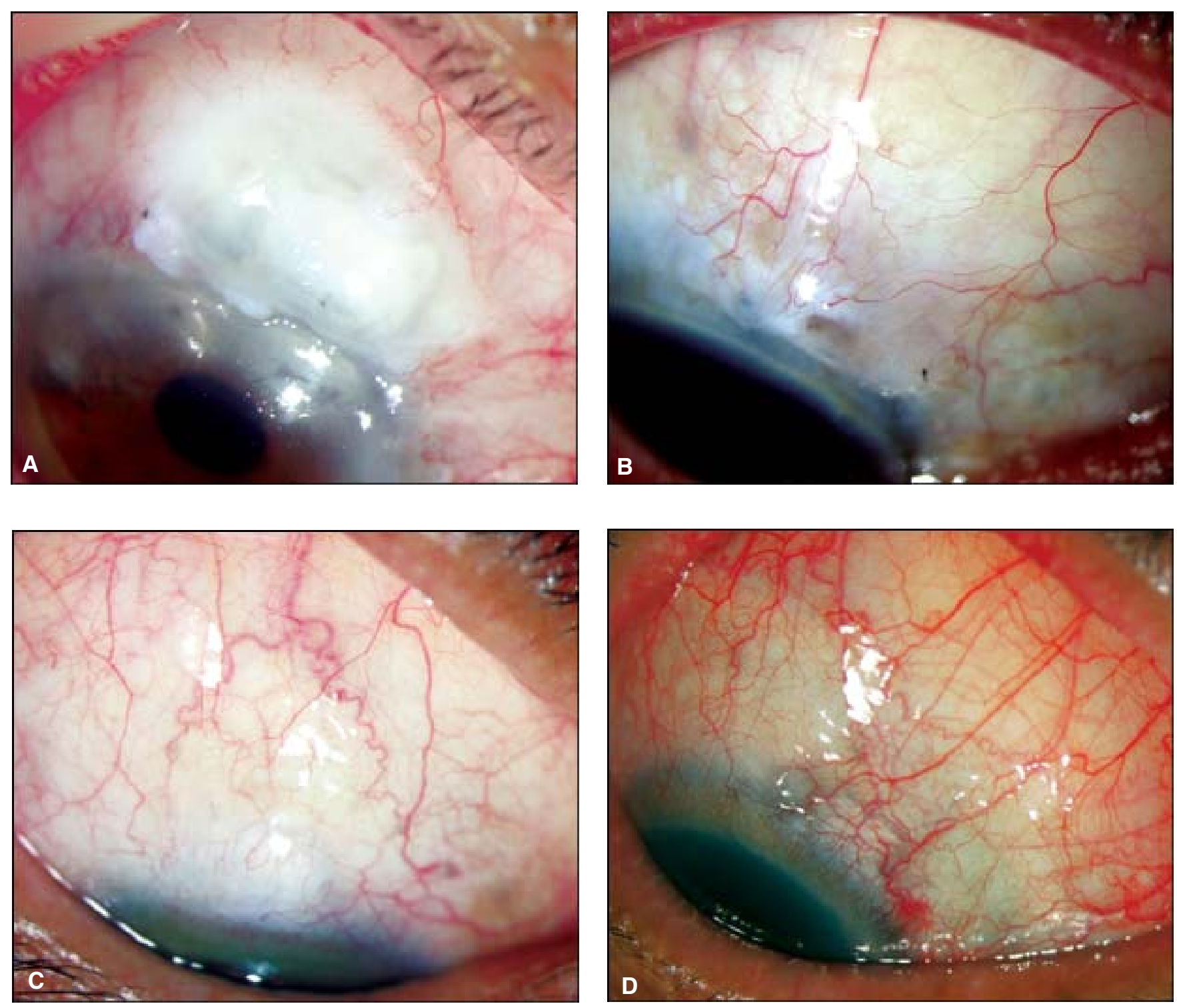

Figs 1A to D: Examples of color mono-photographs of blebs. Using photography, bleb vascularity is easily assessed whereas bleb height and extent are difficult to evaluate. (A) High avascular bleb with a sharply demarcated central portion and a low, diffuse peripheral portion. The peripheral margins of the diffuse portion are difficult to ascertain. The central and peripheral portions of this bleb have different vascularity characteristics. The wall appears thin and cystic centrally; this bleb may be prone to complications such as bleb leak. (B) Low, diffuse bleb spread over a large surface of the sclera with few surface vessels. The surface extent of this bleb cannot be exactly determined from this photograph. This bleb has a favorable morphology and may be less prone to leak due to its low profile and lack of thinning of the wall. (C) Completely flat bleb with no thickening of the conjunctiva. The conjunctiva is apposed to sclera with no intervening episcleral space. This is a poorly formed bleb associated with poor intraocular pressure control. (D) Flat bleb with increased vascularity. There is no conjunctival thickening. Increased vascularity is a poor prognostic factor for bleb function. Lack of conjunctival thickening indicated poor aqueous flow through this shunt

\section{Bleb Classification Systems}

Early efforts at bleb classification featured inconsistent terminology and inexact assessments of the manifold aspects of bleb morphology. Early systems included those proposed by Kronfeld (1969), Migdal and Hitchings (1983) and Picht and Grehn (1998). ${ }^{4,21,22}$ Subsequent bleb grading systems identified and incorporated a graded assessment of various bleb parameters such as vascularity, height, width, microcystic changes, encystment and diffuse/demarcated zones. 
The Indiana Bleb Appearance Grading Scale (IBAGS, 2003) and the Moorfields Bleb Grading System (MBGS, 2004) have been proposed as systems of standardized classification of blebs into groups based on clinical characteristics. ${ }^{23,24}$ The purpose of these systems is to enable a structured framework by which functional outcomes of trabeculectomy may be correlated to bleb structure.

The IBAGS used standards that consisted of slit lamp images for grades of bleb height (H0-H3), extent (E0-E3), vascularity (V0-V4) and leakage with the Siedel test (S0-S2). Each bleb was assigned a grade for each of the features assessed and was reported in the manner $\mathrm{HxExVxSx}$. This system forced the observer to assign one vascularity grade for the whole bleb, when in fact clinicians may find that different areas within any given bleb have different vascularity characteristics.

Reference to photographic standards has been reported in an effort to assess if blebs could be evaluated by a remote observer using telemedicine. ${ }^{25}$ In this study it was found that remote examination of photographs with telemedicine was limited with regards to the grading of bleb height, bleb wall thickness, microcysts and overall bleb morphology. In this respect, bleb imaging may be a valuable tool in documenting these characteristics that are difficult to evaluate in a consistent manner using clinical or photographic assessment.

The MBGS built upon the system used for this telemedicine study and expanded it to include an assessment of vascularity away from the center of the bleb and a way to represent mixedmorphology blebs. In this scheme, central area (1-5), maximal area (1-5), bleb height (1-4) and subconjunctival blood (0-1) were assessed. In addition, three areas of the bleb were graded separately for vascularity, including bleb center conjunctiva, peripheral conjunctiva and non-bleb conjunctiva. Vascularity in each area was assigned a score from 1 to 5 .

A recent study found good inter-observer agreement and clinical reproducibility in the IBAGS and MBGS. ${ }^{26}$

\section{Limitations of Bleb Classification Systems}

These systems are limited, however, in that they do not include a direct assessment of bleb wall thickness, which may have implications for IOP control, bleb leak and bleb-related endophthalmitis. Assessment of bleb wall thickness is challenging both clinically and photographically and may be poorly reproducible between observers. It was reported that some MBGS graders were not able to distinguish between low, diffuse blebs and completely flat blebs on stereo or mono photographs. Diffuse blebs may have such small degrees of thickening of the conjunctiva or separation of the conjunctiva from sclera that are imperceptible on visual examination. Microcystic change of the conjunctiva is also not directly addressed in these studies, being not easily amenable to slit lamp photography. As clinicians are aware, microcysts in the bleb wall are an important indicator of aqueous flow through the bleb; ideally any bleb classification system should include an index to reflect microcystic change.

In these respects imaging may contribute valuable data in bleb characterization, as bleb imaging modalities are able to provide objective evaluation of microcystic change, bleb height and bleb wall thickening. In this way bleb imaging may be an important tool to complement the clinician's assessment of bleb structure.

\section{Ultrasound Biomicroscopy}

Ultrasound biomicroscopy (UBM) is able to provide images of the structures within a bleb. One study classified blebs after trabeculectomy with MMC based on qualitative UBM features, and found certain UBM features that were associated with IOP control. ${ }^{27}$ In this study, four UBM parameters were assessed: intra-bleb reflectivity, visibility of the route under the scleral flap, formation of a cavernous fluid-filled space, and bleb height. The first two of these parameters were found to be associated with the degree of IOP control. Blebs were classified into type L (low-reflective), type H (high-reflective), type E (encapsulated), and type $\mathrm{F}$ (flattened). Type L blebs were associated with favorable outcomes, whereas types $\mathrm{E}$ and $\mathrm{F}$ were seen in cases with poor IOP control.

In another UBM study by MacWhae et al, bleb function was classified as 'good', 'fair' and 'poor' based on UBM findings of a patent filtration pathway from the anterior chamber through to the bleb and the presence or absence of a bleb cavity. Correlating this UBM classification with functional status, it was found that UBM findings had 91\% sensitivity in predicting a functioning bleb and $70 \%$ specificity in predicting a nonfunctioning bleb. ${ }^{28}$ It was found that UBM revealed ciliary body detachment in one eye with good IOP control despite the lack of a clinically apparent bleb. It was also suggested that UBM might assist in determining the site and cause of failed filtration in eyes with poor IOP control.

In one study UBM was used to evaluate blebs after laser suture lysis. ${ }^{29}$ This study reached similar conclusions with other UBM studies, i.e. that UBM images of eyes with good IOP control were characterized by better visibility of the route under the scleral flap and a low reflectivity inside the bleb.

UBM imaging of trabeculectomy blebs is limited in that contact is required with the eye. This is undesirable in the postoperative setting, in which concerns of infection are paramount. In addition, UBM imaging may cause significant discomfort to patients due to the use of the eyecup in contact with the globe. In one study, a number of patients were excluded, as patients could not tolerate the discomfort associated with the use of the eyecup. ${ }^{28}$ 


\section{Anterior Segment Optical Coherence Tomography}

The principles of optical coherence tomography (OCT) and its application in imaging the anterior segment are well described. ${ }^{30,31}$ In the context of glaucoma, the anterior segment OCT (ASOCT) has been used in the assessment of the iridocorneal angle. ${ }^{32}$ ASOCT imaging is faster than UBM and also eliminates the need for a water bath and eyecup in contact with the globe and is therefore a more comfortable procedure for the patient.

An early report by Savini et al described the use of standard posterior segment OCT in visualizing blebs. ${ }^{33}$ Blebs were classified into categories according to OCT pattern: type A (featuring a thick wall and a single large fluid-filled space), type $\mathrm{B}$ (featuring a thin wall and multiple large fluid-filled spaces) and type $\mathrm{C}$ (featuring multiple, irregular and flattened fluidfilled spaces). Fluid-filled spaces were observed in most of the blebs with good IOP control. This study was also useful in showing the ability of OCT imaging to show distinct morphological features in eyes treated with MMC compared to those with no MMC application.

Muller et al in 2006 reported the use of slit-lamp adapted $1310 \mathrm{~nm}$ OCT as an in vivo imaging device for filtering blebs and deep sclerectomies in 28 patients. ${ }^{34}$ OCT using this wavelength is suitable for anterior segment imaging. OCT was able to demonstrate the internal structure of the bleb and the deep sclerectomy site. Functioning blebs showed a low OCT signal, small fluid-filled cysts, superficial microcystic layer, and a slack internal texture. Nonfunctioning blebs delivered a high OCT signal, no or few cysts, and a dense internal texture. The authors suggested that high internal reflectivity indicated scarring of the bleb, and proposed OCT as a way to assess the postoperative healing process with the possibility of early intervention in cases of impending scarring.

This study was followed by a number of reports of the use of ASOCT in imaging trabeculectomy blebs. Leung et al imaged 14 post-trabeculectomy eyes with ASOCT, describing the different OCT features of bleb morphology such as bleb wall thickness, subconjunctival fluid collections, suprascleral fluid space, scleral flap thickness, intrableb intensity and the route under the scleral flap. These features were correlated with bleb type classified by slit lamp appearance (diffuse, cystic, encapsulated and flattened). ${ }^{35}$

Our group in 2007 reported a prospective study of ASOCT imaging of blebs in relation to function. ${ }^{36}$ Seventy-eight blebs in 55 patients were imaged, and blebs were classified as successful or failed based on IOP criteria using $18 \mathrm{mmHg}$ as a cutoff value. ASOCT was able to identify total bleb height, the bleb cavity, bleb wall thickness, tangential and radial dimensions of the bleb, scleral flap thickness and position, and patency of the internal keratotrabeculectomy ostium. Using ASOCT images, blebs were classified into high, moderate-height and low blebs based on semi-quantitative comparison to scleral thickness. ASOCT grading of bleb height correlated well with clinical grading of bleb height by a masked observer according to the MBGS scale.

In this study the majority of successful blebs displayed thickening of the bleb wall and were mostly high or moderateheight blebs. The term 'thickening' was used in reference to the linear dimension of height rather than to the intensity of OCT signal, which may indicate the density of tissue in the bleb wall. Microcysts were seen in a number of successful blebs. Failed blebs were mostly low and thickening of the bleb wall was typically absent. In most failed cases, ASOCT was able to suggest the structural cause of failure such as internal ostial occlusion, apposition of conjunctiva-episclera to sclera or apposition of the scleral flap to its bed.

Our study also correlated ASOCT findings to functional outcome by describing unique morphological features of successful and failed blebs. The findings of this study also suggested that ASOCT imaging of blebs may be used in conjunction with the MBGS to address the latter's limitations in grading bleb height, wall thickness and microcystic changes.

In a subsequent study we further described the use of ASOCT in characterizing the morphological change induced by laser suture lysis (LSL), finding features associated with success to be present after LSL was undertaken in failed blebs. ${ }^{37}$

\section{In Vivo Confocal Microscopy}

Labbé et al in 2005 reported a retrospective analysis of bleb structure at the tissue level using an in vivo confocal microscope (IVCM) in 17 eyes. ${ }^{38}$ IVCM images were analyzed for the number of intraepithelial microcysts, density of subepithelial connective tissue, presence of blood vessels, and encapsulation. In this study all functioning blebs had numerous intraepithelial microcysts, whereas all nonfunctioning blebs had none or few. Subepithelial connective tissue was widely spaced in all functioning blebs but dense in most nonfunctioning blebs. This report was unique in providing images of blebs at the tissue level, and showed microcystic change more clearly than ASOCT imaging. IVCM may possibly be able to show the subtle effects of antimetabolites on tissue characteristics bleb structure and may be useful in assessing response to wound healing modulation in any given bleb.

\section{Impression Cytology}

In a further recent study by Amar et al, impression cytology was used in conjunction with IVCM to identify cellular characteristics that corresponded to IVCM appearance and bleb function. ${ }^{39}$ Goblet cells were identified using mouse anti-M1 immunoglobulin $\mathrm{G}$, recognizing products of the MUC5AC gene 
corresponding to soluble mucins of goblet cells. The authors found the presence of numerous goblet cells that showed weak or no MUC5AC immunostaining at the surface of the functioning blebs, as opposed to highly stained goblet cells outside the limit of the blebs. These cells seemed to correspond to the microcysts observed with in vivo confocal microscopy, and were observed only in very low numbers on the surface of nonfunctioning blebs. The authors suggest that the numerous goblet cells that corresponded to the microcysts observed with IVCM were probably channels for the passage of aqueous humor. It was hypothesized that these goblet cells mostly contained aqueous humor instead of gel-forming mucins, which are highly hydrophilic, and may in this way favor aqueous transconjunctival outflow. Eyes in which MMC were used differed from non-MMC eyes in that a higher number of inflammatory cells were found in the former.

\section{Vascular Assessment of Blebs}

Alsagoff and coworkers in 2001 described the use of indocyanine green (ICG) for angiography of the anterior segment to characterize conjunctival and episcleral vasculature changes in 10 blebs. ${ }^{40}$ The study showed that with ICG, the anterior segment vessels and deep episcleral veins were well delineated and that there was reduced vascularity over the blebs along with vascular anastomoses along the perimeter of avascular blebs. The findings of report suggest that ICG angiography might be able to assess vascular changes after trabeculectomy more accurately than slit-lamp assessment. Angiography is an invasive procedure with a small but definite morbidity, and may not be practical in the routine assessment of patients after trabeculectomy. It may be useful, however, in the research setting in evaluating vascular responses to wound healing modulation or different surgical techniques.

\section{Potential Role of Bleb Classification}

Bleb imaging in relation to function is pertinent in assessing outcomes of surgery with the use of new adjuncts that have been reported such as human antibody to transforming growth factor b2, CAT-152. ${ }^{41}$ It may also have a role in evaluating the filtration function of eyes treated with new surgical methods such as nonpenetrating sclerostomy. ${ }^{42}$ The advent of adjuncts to modulate wound healing in trabeculectomy, and new surgical techniques to improve success, demands a comprehensive way of describing blebs in an effort to evaluate structural and functional outcomes of trabeculectomy surgery.

\section{CONCLUSION}

Blebs have been characterized using a variety of modalities including clinical and photographic assessment, UBM, OCT, IVCM, impression cytology, and ICG angiography. Clinical grading and ASOCT imaging may provide complementary information on gross structure and may, in combination, be able to characterize bleb height, extent, wall thickness and surface vascularity. IVCM may provide detailed information about microcystic changes and other tissue characteristics in the bleb, which are relevant to functional status. Cellular changes such as alteration in number and type of goblet cells may be evaluated using impression cytology. Finally, ICG angiography may be used to delineate vascular patterns in and around the bleb.

The complete characterization of any bleb involves the description and evaluation of a multitude of aspects including gross structure, microscopic structure, cellular changes and vascular alterations. A comprehensive classification system may involve the synthesis of data obtained by a combination of multiple imaging and assessment modalities in order to reach a complete and thorough characterization of blebs.

\section{REFERENCES}

1. Cairns JE. Trabeculectomy. Am J Ophthalmol 1968;66:673-79.

2. Vogel R, Crick RP, Newson RB, et al. Association between intraocular pressure and loss of visual field in chronic simple glaucoma. Br J Ophthalmol 1990;74:3-6.

3. O’Brien C, Schwartz B, Takamoto T, Wu DC. Intraocular pressure and the rate of visual field loss in chronic open-angle glaucoma. Am J Ophthalmol 1991;111:491-500.

4. Picht G, Grehn F. Classification of filtering blebs in trabeculectomy: biomicroscopy and functionality. Curr Op Ophthalmol 1998;9:2-8.

5. Vesti E. Filtering blebs: follow-up of trabeculectomy. Ophthalmic Surg Lasers 1993;24:249-55.

6. Singleton BJ. Management of the failing glaucoma filter. Surg Rev 1996;27,445-51.

7. Picht G, Grehn F. Classification of filtering blebs: a correlation to outcome 3 months after trabeculectomy (ARVO abstract). Invest Ophthalmol Vis Sci 1997;38:1060.

8. Sacu S, Rainer G, Findl O, et al. Correlation between the early morphological appearance of filtering blebs and outcome of trabeculectomy with mitomycin C. J Glaucoma 2003;12:430-5.

9. Palmer SS. Mitomycin as adjunct chemotherapy with trabeculectomy. Ophthalmology 1991;98:317-21.

10. Kitazawa Y, Kawase K, Matsushita H, Minobe $M$. Trabeculectomy with mitomycin. A comparative study with fluorouracil. Arch Ophthalmol 1991;109:1693-8.

11. Singh K, Mehta K, Shaikh NM, et al. Trabeculectomy with intraoperative mitomycin $\mathrm{C}$ versus 5-fluorouracil. Prospective randomized clinical trial. Ophthalmology 2000;107:2305-9.

12. Shields MB, Scroggs MW, Sloop CM, Simmons RB. Clinical and histopathologic observations concerning hypotony after trabeculectomy with adjunctive mitomycin C. Am J Ophthalmol 1993;116:673-83.

13. Hu C, Matsuo H, Tomita G, et al. Clinical characteristics and leakage of functioning blebs after trabeculectomy with mitomycin-C in primary glaucoma patients. Ophthalmology 2003;110:345-52. 
14. Azuara-Blanco A, Katz LJ. Dysfunctional filtering blebs. Surv Ophthalmol 1998;43:93-126.

15. Nuyts RMMA, Felten PC, Pels E, et al. Histopathologic effects of mitomycin $\mathrm{C}$ after trabeculectomy in human glaucomatous eyes with persistent hypotony. Am J Ophthalmol 1994;118:22537.

16. Soltau JB, Rothman RF, Budenz DL, et al. Risk factors for glaucoma filtering bleb infections. Arch Ophthalmol 2000;118:338-42.

17. Poulsen EJ, Allingham RR. Characteristics and risk factors of infections after glaucoma filtering surgery. J Glaucoma 2000;9:438-43.

18. Greenfield DS, Suñer IJ, Miller MP, et al. Endophthalmitis after filtering surgery with mitomycin. Arch Ophthalmol 1996;114:943-9.

19. Lehmann OJ, Bunce C, Matheson MM, et al. Risk factors for development of post-trabeculectomy endophthalmitis. Br J Ophthalmol 2000;84:1349-53.

20. DeBry PW, Perkins TW, Heatly G, et al. Incidence of late-onset bleb-related complications following trabeculectomy with mitomycin. Arch Ophthalmol 2002;120:297-300.

21. Kronfeld PC. Functional characteristics of surgically produced outflow channels. Trans Am Acad Ophthalmol Otolarynglol 1969;73,177-93.

22. Migdal C, Hitchings R. The developing bleb: effect of topical antiprostaglandins on the outcome of glaucoma fistulising surgery. Br J Ophthalmol 1983;67:655-60.

23. Cantor LB, Mantravadi A, WuDunn D, et al. Morphologic classification of filtering blebs after glaucoma filtration surgery: the Indiana Bleb Appearance Grading Scale. J Glaucoma 2003;12:266-71.

24. Wells AP, Crowston JG, Marks J. A pilot study of a system for grading drainage blebs after glaucoma surgery. J Glaucoma 2004;13(6):454-60.

25. Crowston JG, Kirwan JF, Wells A, et al. Evaluating clinical signs in trabeculectomized eyes. Eye 2004;18(3):299-303.

26. Wells AP, Ashraff NN, Hall RC, et al. Comparison of two clinical bleb grading systems. Ophthalmology 2006;113:77-83.

27. Yamamoto T, Sakuma T, Kitazawa Y. An ultrasound biomicroscopic study of filtering blebs after mitomycin C trabeculectomy. Ophthalmology 1995;102:1770-76.

28. McWhae JA, Crichton ACS. The use of ultrasound biomicroscopy following trabeculectomy. Can J Ophthalmol 1996;31(4):187-91.

29. Avitabile T, Russo V, Uva MG, et al. Ultrasound biomicroscopic evaluation of filtering blebs after laser suture lysis trabeculectomy. Ophthalmologica 1998;21 (suppl 1):17-21.

30. Huang D, Swanson EA, Lin CP, et al. Optical coherence tomography. Science 1991;254:1178-81.

31. Radhakrishnan S, Rollins AM, Roth JE, et al. Real-time optical coherence tomography of the anterior segment at $1310 \mathrm{~nm}$. Arch Ophthalmol 2001;119:1179-85.

32. Radhakrishnan S, Goldsmith J, Huang D, et al. Comparison of optical coherence tomography and ultrasound biomicroscopy for detection of narrow anterior chamber angles. Arch Ophthalmol 2005;123:1053-59.
33. Savini G, Zanini M, Barboni P. Filtering blebs imaging by optical coherence tomography. Clin Experiment Ophthalmol 2005;33(5):483-9.

34. Muller M, Hoerauf H, Geerling G, et al. Filtering bleb evaluation with slit-lamp-adapted 1310-nm optical coherence tomography. Curr Eye Res 2006;31(11):909-15.

35. Leung CK, Yick DW, Kwong YY, et al. Analysis of bleb morphology after trabeculectomy with Visante anterior segment optical coherence tomography. Br J Ophthalmol 2007;91(3):3404. Epub 2006 Sep 27.

36. Singh M, Chew PTK, Friedman DS, et al. Imaging of trabeculectomy blebs using anterior segment optical coherence tomography. Ophthalmology 2007;114(1):47-53. Epub 2006 Oct 27.

37. Singh M, Aung T, Friedman DS, et al. Anterior segment optical coherence tomography imaging of trabeculectomy blebs before and after laser suture lysis. Am J Ophthalmol 2007;143(5):8735.

38. Labbé A, Dupas B, Hamard P, Baudouin C. In vivo confocal microscopy study of blebs after filtering surgery. Ophthalmology 2005;112:1979-86

39. Amar N, Labbé A, Hamard P, et al. Filtering blebs and aqueous pathway: an immunocytological and in vivo confocal microscopy study. Ophthalmology 2008 (in press).

40. Alsagoff Z, Chew PT, Chee CK, et al. Indocyanine green anterior segment angiography for studying conjunctival vascular changes after trabeculectomy. Clin Experiment Ophthalmol 2001; 29(1):22-6.

41. Khaw P, Grehn F, Hollo G, CAT-152 0102 Trabeculectomy Study Group. A phase III study of subconjunctival human antiTGF-beta2 monoclonal antibody (CAT-152) to prevent scarring following first-time trabeculectomy. Ophthalmology 2007;114(10):1822-30.

42. Shaarawy $\mathrm{T}, \mathrm{Wu} \mathrm{R}$, Mermoud A, et al. Influence of nonpenetrating glaucoma surgery on aqueous outflow facility in isolated porcine eyes. Br J Ophthalmol 2004;88:950-52.

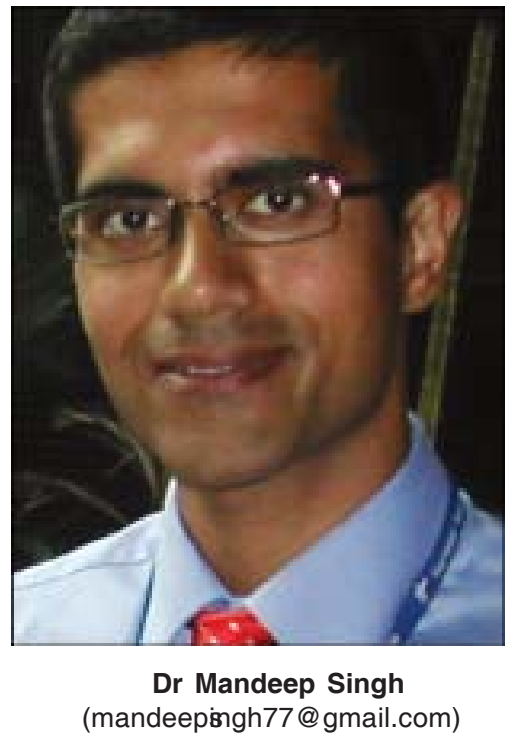




\title{
Submitting a paper/video to Journal of Current Glaucoma Practice (Glaucoma Review Journal with DVD)
}

\begin{abstract}
INSTRUCTION TO AUTHORS
Journal of current glaucoma practice is a glaucoma review journal with video assisted teaching. It publishes invited review articles on basic and clinical sciences in Glaucoma and videos related to glaucoma diagnostics or surgery. Original research material can also be integrated into a review article. Articles should be submitted only by individuals with experience and expertise in the topic that they are reviewing. Videos can be submitted by any glaucoma specialist and may be incorporated into the journal DVD after peer review.

Before preparing a manuscript, the author should submit a detailed outline of the proposed article to the Editor-in-Chief to assure that the material is appropriate and that no similar article is in preparation. Please allow 2 to 4 weeks for a response.
\end{abstract}

Please do not hesitate to contact the Editorial Office if you have any questions.

\section{THE REVIEW}

Your review can be up to 2500 words in length and should highlight and discuss all interesting developments in the subject, as reflected in the recent literature. In addition to describing recent trends, you can give a synopsis of your own opinions of the topics discussed and suggest a preferred practice pattern.

\section{MANUSCRIPT FORMAT}

The review must be double-spaced and a maximum of 2500 words in length (excluding references).

\section{Review structure}

The review must contain the following:

Cover page: Stating the title, authors and their affiliations, and full contact details for the corresponding author (including phone number and e-mail address).

Introduction: This should be a paragraph of 50-100 words outlining the scope of the review and mentioning any earlier work which will place the review in context.

Text of review: Includes headings and titled paragraphs to subdivide the text. Ensure that at least one sentence divides each heading (i.e. do not have a subheading directly beneath a full heading).

Conclusion: A paragraph of 50-100 words drawing together the implications of the review topic and, if appropriate, giving suggestions for future research.

Acknowledgements: Of professional colleagues and funding bodies only.

Reference section: References should be in numerical sequence (Vancouver style), include the first three authors, or all authors if there are six or fewer.

Figure titles and legends: Must be provided for all figures.

Figures and tables: Must be cited in text.

Figures and tables must be

Original whenever possible

Clearly marked as "original" or "previously published" upon submission

Accompanied by full source details when not original

Figures should not be embedded within the text but should be submitted as separate files. Figure legends should be included in the main body of the text following the references.

References cited in figures or tables must be numbered in sequence, according to the position of the first text citation of the figure or table.

\section{Illustrations}

Original ideas for explanatory diagrams are welcomed. Electronic artwork should be submitted in JPEG, TIFF, EPS or PPT formats. Please state clearly whether figures/tables have been published previously.

Color figures: There is a no charge to authors if you wish your figures to be produced in color. 


\section{DISCLOSURES}

Authors should include all relevant information regarding Conflict of Interest and Sponsorship Statements.

\section{SUBMITTING YOUR PAPER}

Manuscripts should be submitted by e-mail at currentglaucomapractice@gmail.com

If you have any query or suggestion, please do not hesitate to contact:

Editor-in Chief

Tanuj Dada, MD, Dr Rajendra Prasad Centre for Ophthalmic Sciences, All India Institute of Medical Sciences, New Delhi, India e-mail: tanujdada@ hotmail.com

The manuscript file should include the text, references, structured abstract, keywords, figure legends and author address. Figures and tables should be submitted as separate files.

References cited in figures or tables must be numbered in sequence, according to the position of the first text citation of the figure or table. Unpublished data, submitted manuscripts and personal communications must be referenced in the text only as follows:

Personal communication: (Author A, personal communication).

Submitted paper: (Author A, Author B, unpublished data).

You are responsible for the accuracy of the references and for obtaining permission to use personal communications.

\section{REFERENCE FORMAT}

In the text, reference citations should be typed in superscript.

Please list the first three authors for each reference and then et al, unless there are six authors or fewer, in which case all authors should be listed. Provide full reference details (author(s), title, journal, year, volume, pages).

Journal names should be abbreviated as in the Index Medicus.

References should be structured as follows:

Journal:

Author A, Author B, Author C. Title of the paper. Journal Abbreviation 2000; 4:25-27.

With annotation describing importance of reference, if bulleted

Book:

Author A, Author B, Author C. Title of the book section. In: Book name. Edition number. Edited by Editor A, Editor B, Editor C (editors). Location of Publisher: Publisher; 2000. pp. 25-27.

Copyright transfer form with article title and signature of all authors needs to be mailed or faxed during submission of the article.

\section{SUBMISSION OF VIDEOS}

The journal accepts videos demonstrating surgical techniques and newer diagnostic techniques. Videos for the CD/DVD should be edited with an audio commentary or a text overlay and submitted in the following formats: .mpg/.wmv/.dat The CD/DVD with video may be directly sent to the journal editorial office (address given below).

Video should be accompanied by

Cover page: Stating the title, authors and their affiliations, and full contact details for the corresponding author (including phone number and e-mail address).

A small summary of the procedure being shown in about 150 words should be included.

Video should be accompanied by a moving legend describing the procedure with or without an audio in English language.

ADDRESS OF THE JOURNAL OFFICE FOR SUBMISSION OF VCD OR DVD VIDEOS:

Jaypee Brothers Medical Publishers (P) Ltd., 23/23B EMCA House, Ansari Road, Daryaganj, New Delhi - 110002, India

Phones: +91-11-23272143, +91-11-23272703, +91-11-23282021, +91-11-23245672

Rel: 32558559 Fax: +91-11-23276490, +91-11-23245683

e-mail: jaypee@jaypeebrothers.com 


\section{SUBSCRIPTION INFORMATION}

Annual subscription:

Individual: Rs. 1600.00

$\$ 130.00$

£ 75.00

Institutional: Rs. 3000.00

$\$ 160.00$

$£ \quad 100.00$ (national)

(international)

(international)

(national)

(international)

(international)
Subscription can be sent to

M/s Jaypee Brothers Medical Publishers (P) Ltd

Journals Department

Jaypee Brothers Medical Publishers (P) Ltd

4838/24 Ansari Road, Daryaganj

New Delhi 110002 India

Phone: +91-11-43574357

This journal is published thrice in a year, i.e. January, May and September, every year. Dollar rates apply to subscribers in all the countries except the UK and the Republic of Ireland where the pound/sterling price applies. All subscriptions are payable in advance and all the rates include postage. Journals are sent by air to all the countries except Indian subcontinent. Subscriptions are on an annual basis, i.e. from January to December. Payment will be made by sterling cheque, dollar cheque, credit card or directly through our bank account at the following address:

1. Our banker's name:

Canara Bank, Netaji Subhash Marg

Darya Ganj, New Delhi 110002

2. Telephone No:

011-23273015, 011-23273849

3. Fax No:

011-23255606

4. Telex Number:

3166291

5. Our Current A/c No:

3828

6. Amount to be transferred

JAYPEE BROTHERS MEDICAL in the name of:

7. Swift code No:

PUBLISHERS (P) LTD., NEW DELHI

CNRB IN BB DFM

For further queries please do not hesitate to contact MR TARUN DUNEJA at e-mail: tarunduneja@gmail.com

\section{ADVERTISEMENT RATES}

(For the Print Issues)

Page

Single issue

Back cover-colour

\begin{tabular}{lll}
\hline Rs. 25,000 & $\$ 625.00$ & ð 450 \\
Rs. 20,000 & $\$ 500.00$ & ð 350 \\
Rs. 15,000 & $\$ 375.00$ & ð 270 \\
Rs. 12,500 & $\$ 300.00$ & ð 220 \\
Rs. 10,000 & $\$ 250.00$ & ठ 175
\end{tabular}

\begin{tabular}{|c|c|c|}
\hline \multicolumn{3}{|c|}{ Three issues } \\
\hline Rs. 60,000 & $\$ 1600.00$ & ð 1050 \\
\hline Rs. 50,000 & $\$ 1400.00$ & ð 890 \\
\hline Rs. 40,000 & $\$ 1100.00$ & ð 700 \\
\hline Rs. 34,000 & $\$ 850.00$ & ð 600 \\
\hline Rs. 27,000 & $\$ 675.00$ & ð 480 \\
\hline
\end{tabular}

*First page, page facing editorial board, page facing table of contents.

Cover page advertisements not available for a single issue.

Technical Details

Paper size

$8.5 \times 11.5$ inches

Print size

$7 \times 10$ inches

Digital file format

EPS on CD (at 300 dpi resolution)

Printed on art paper using offset printing.

Schedule

Issues are published in the months of January, May and September.

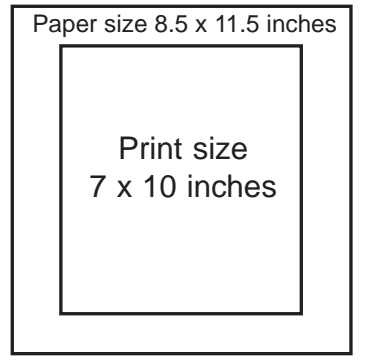

Advertisement material along with purchase order and payment should reach us at least four weeks prior to the scheduled print date.

\section{Payment Details}

- Payment should favour "Jaypee Brothers Medical Publishers (P) Ltd." and should be payable at New Delhi, India.

- Payment to be done at the time of submitting the advertisement material/booking the advertisement. Please send your advertisement request, payment and advertisement material to the address given above. Editorial board reserves the right to accept or decline the advertisement. 


\section{The Ahmed $\mathbf{d}^{\mathrm{Tm}}$ Elaucoma Valve}

Leading the way in Advanced Claucoma Drainage Technology

\section{Flexible Plate ${ }^{\mathrm{TM}} \boldsymbol{\varepsilon}$} Human Allograft Tissue

Human

Allograft Tissue Processed Sclera \& Pericardium
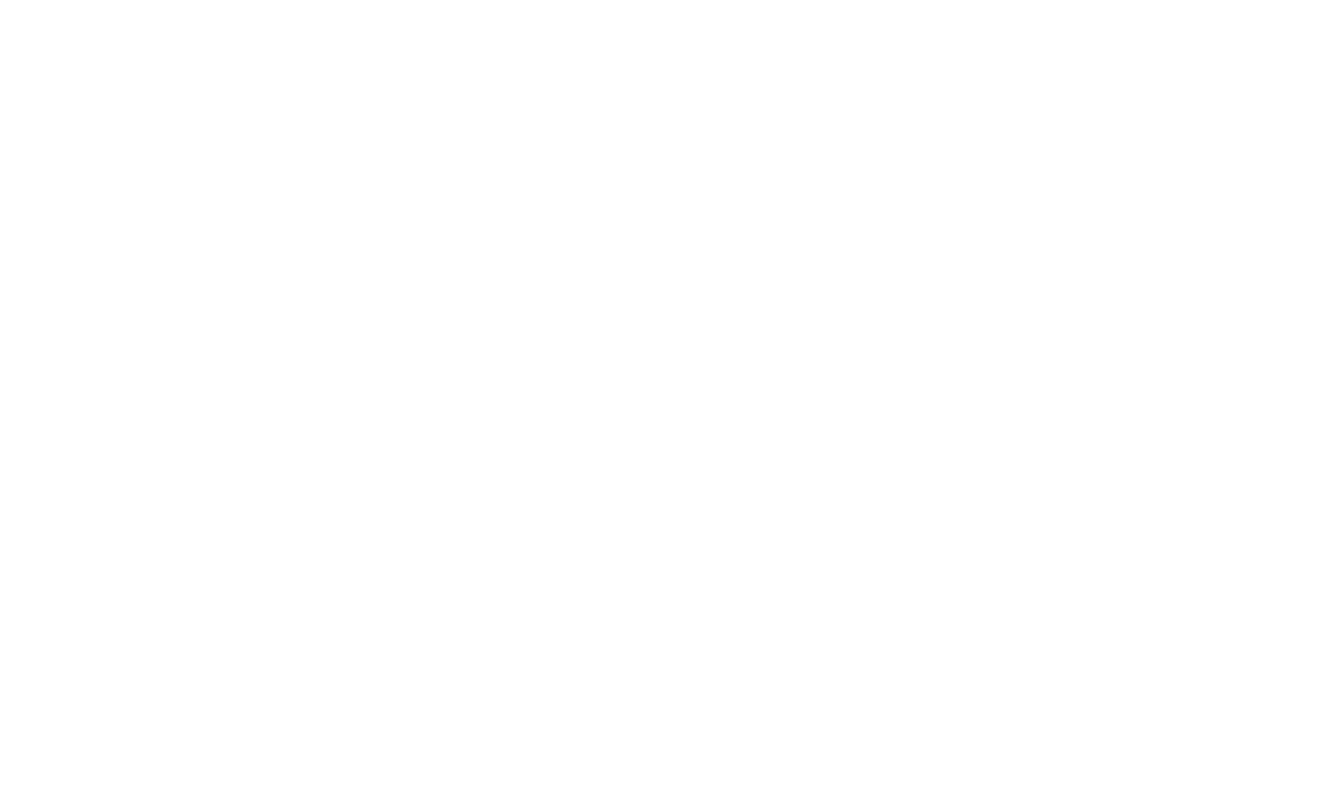

Model FP7 Flexible Plate ${ }^{\mathrm{m}}$ Surface area: $184.00 \mathrm{~mm}^{2}$

CARPEL MEDICOS PVT. LTD.

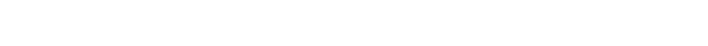

A 701 , Indraprastha Villa

Sector-61 NOIDA-201301

NCR-DELHI

Ph. 120-4313928 - Fax. 0120-4255615

Cell. 9811270823,9311360823
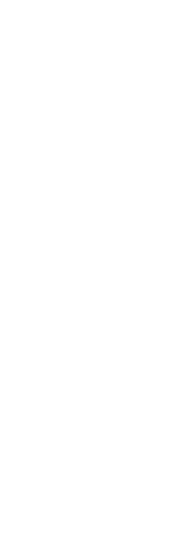

Cell, 9811270923,931136023 

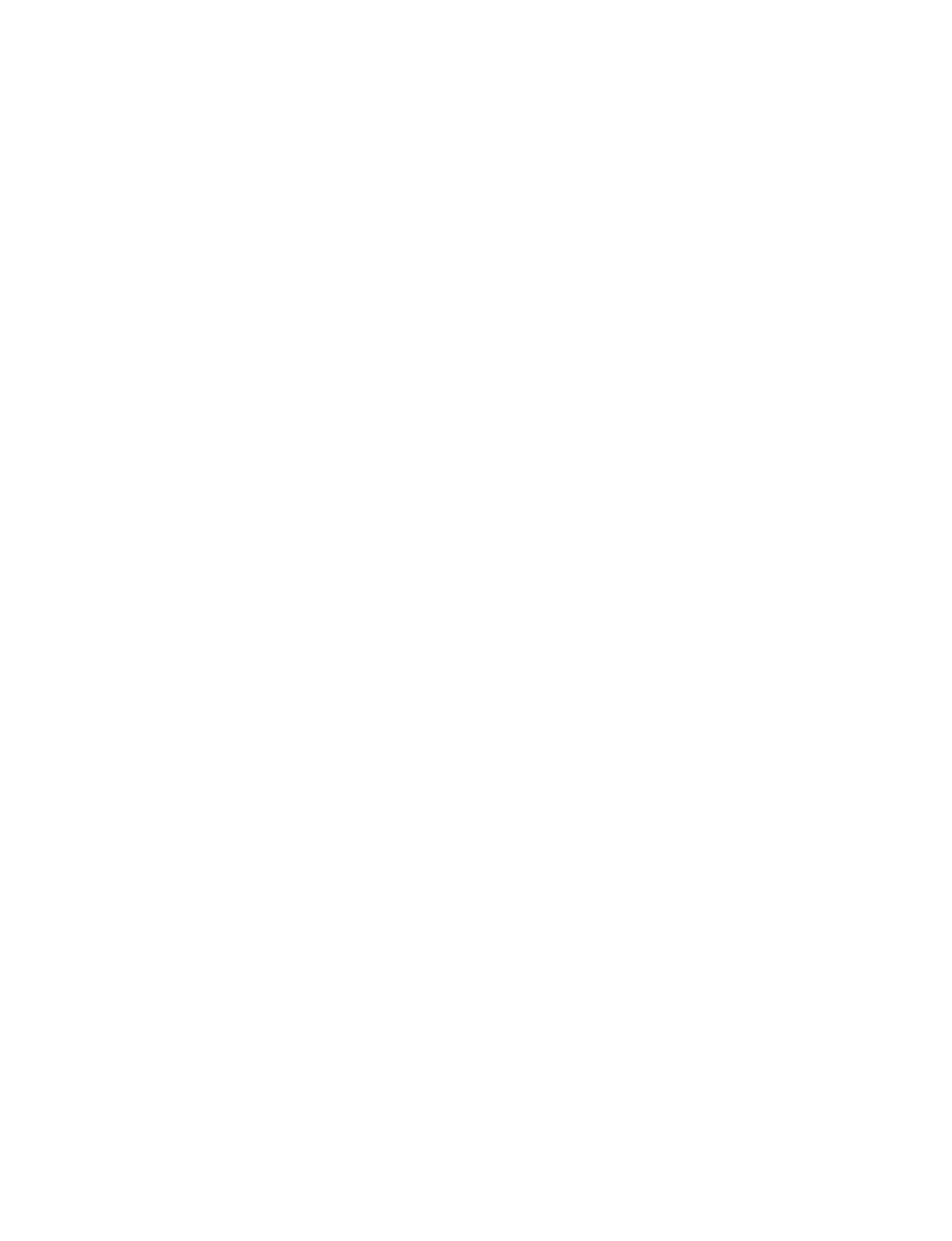
ALL THIS...

AND A SLIT LAMP TOO.

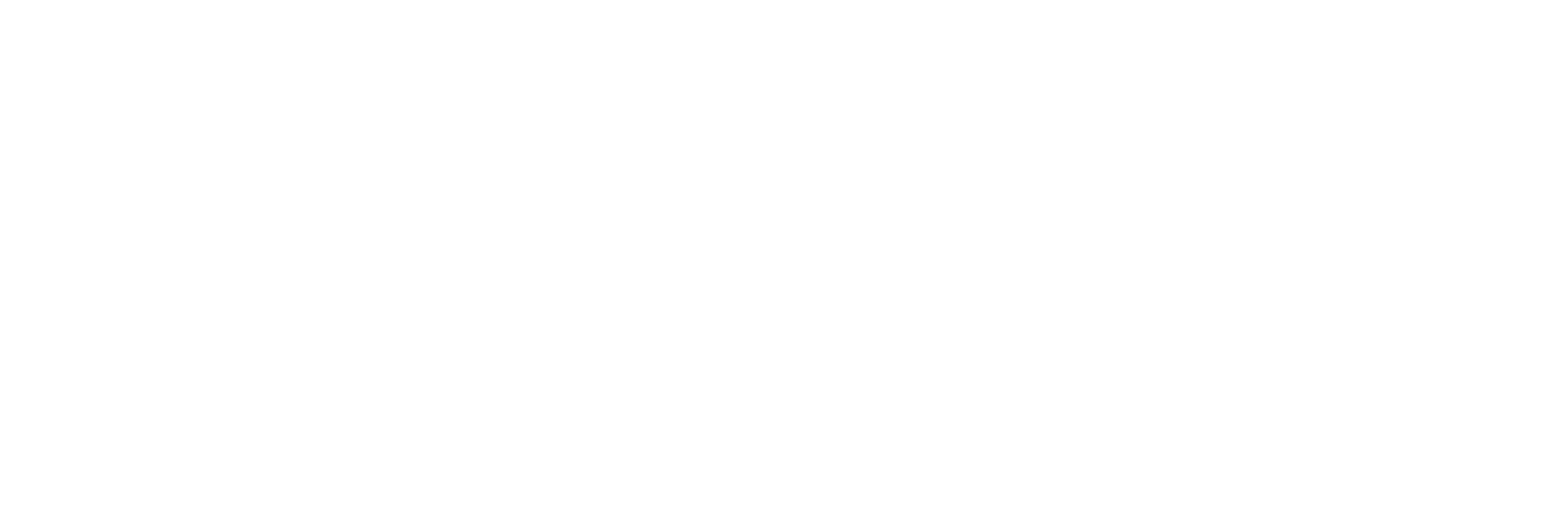

\section{바-ㅁㄷㄴ}

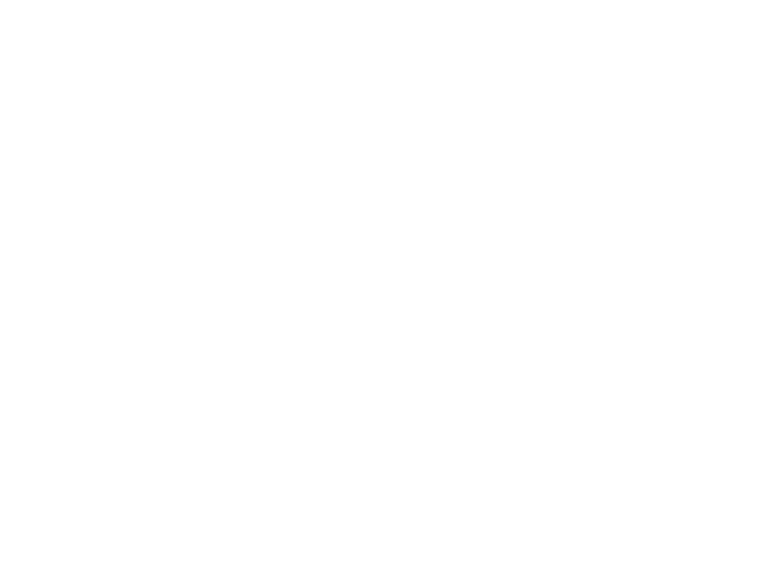

For further information please call: +496221 6463-0

www. HeidelbergEngineering.com
The Heidelberg Engineering slit lamp OCT (SL-OCT) is a true multi-functional solution that combines sit lamp, anterior segment OCT, and external ocular photography in one powerful device.

The SL-OCT with Digital Gonioscopy allows convenient non-contact examination of angle-closure glaucoma in darkness and without distortion of the cornea.

\section{Slit lamp-OCT}

Numerous applications:

n Glaucoma screening (PACG)

- Digital goniometry

I Pachymetry

auto-Biometry

4 Anterior chamber measurement

E External digital photography

\section{HEIDELBETE

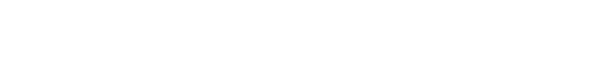

